\title{
Evaluation of Pesticide Effects on Microbial Communities in a Paddy Soil Comparing with That Caused by Soil Flooding
}

\author{
Kazuhito IтOH, ${ }^{*}$ Takahiro IKUSHIMA, Kousuke SUYAMA and Hiroki Yamamoto \\ Faculty of Life and Environmental Science, Shimane University, Matsue, Shimane 690-8504, Japan
}

(August 12, 2002; Accepted November 26, 2002)

\begin{abstract}
The Effect of a herbicide Zark D51 (ZD, $4.5 \%$ daimuron and $0.51 \%$ bensulfuron methyl) powder and a fungicide Fuji-One Moncut (MC, $12.0 \%$ isoprothiolane and $7.0 \%$ flutolanil) powder on microbial communities in a paddy soil was examined in laboratory experiments using Biolog GN plates. ZD had little effect on the soil microbial communities at the recommended and 50 times the recommended rate. MC showed no significant effect at the recommended rate, however, the 50-fold application caused changes in the microbial communities for at least four weeks although the activity to utilize carbon as substrate determined by color development in the Biolog plate had recovered by that time. For assessing the significance of the change in microbial communities, the magnitude of the change caused by MC was compared with that caused by soil flooding which had been shown to be the most influential environmental parameter acting on the microbial communities in paddy soils. At one week after the application of 10 times the recommended dose of $\mathrm{MC}$, the microbial communities could be differentiated by the soil flooding, but not the application of MC. On the other hand, at four weeks after the application, the microbial communities could be distinctly separated by the MC application beyond the magnitude caused by the flooding. A reasonable approach is proposed for assessing the effects of pesticide on soil microbial communities.
\end{abstract}

Key words: paddy soil, Biolog, microbial community structure, carbon substrate utilizing activity, risk assessment of pesticide.

\section{INTRODUCTION}

Assessing the side-effects of pesticides on soil microbial ecosystems is important to maintain soil fertility and to prevent critical damage to agricultural ecosystems. ${ }^{1,2)}$ As soil microbial parameters, such as microbial population, biomass, activity, and community structure, could be affected by natural stresses and fluctuate in the environment, the side-effects caused by pesticides should be evaluated by comparing them with those caused by natural stresses. ${ }^{3)}$ For this purpose, we monitored the range of natural fluctuations in soil microbial populations, biomass, activities, and community structures in rice paddy fields to establish criteria to be used as a reference for risk assessment of pesticides in soil microbial ecosystems. ${ }^{4-6)}$

In our previous study, natural fluctuations of soil microbial communities in two rice paddy fields were monitored using Biolog GN plates for almost two years, and it was revealed that the communities were grouped into three clus-

\footnotetext{
* To whom correspondence should be addressed.

E-mail: itohkz@life.shimane-u.ac.jp
}

ters based on the season, and that the same microbial communities were reestablished in the same season for two successive years in both soils. Reductive soil conditions and low soil temperature seemed to be influential in the establishment of the microbial communities. Based on the information obtained from the study, we proposed that the deviation from the natural fluctuations in the microbial communities caused by the pesticides should be considered significant.

In this study, laboratory experiments were carried out to evaluate the effect of pesticides on microbial communities in a rice paddy soil. The magnitude of the effects caused by the pesticides was compared with that caused by soil flooding, which was the most influential stress on the soil microbial communities in rice paddy fields. ${ }^{6}$

\section{MATERIALS AND METHODS}

\section{Soil Sample}

Soil samples used were collected from a paddy field in Matsue, Shimane prefecture, Japan, where neither pesticides nor chemical fertilizers had been used for more than 7 years. $^{4}$ The surface layer $\left(0^{-}-2 \mathrm{~cm}\right)$ of the paddy soil was 
passed through a $2 \mathrm{~mm}$ sieve, then supplemented with distilled water to achieve a final water content of $50-60 \%$, if necessary, and used for the experiment. The physicochemical characteristics of the soil were presented in a previous paper. ${ }^{4}$

\section{Pesticide Treatment and Incubation of Soil Sample}

A formulation of herbicide, Zark D51 powder (ZD), containing $4.5 \%$ daimuron [1-( $\alpha, \alpha$-dimethylbenzyl)-3-p-tolylurea] and $0.51 \%$ bensulfuron-methyl [methyl $\alpha$-(4,6-dimethoxypyrimidin-2-ylcarbamoylsulfamoyl)-o-toluate], and a formulation of fungicide, Fuji-One Moncut powder (MC), containing $12.0 \%$ isoprothiolane (diisopropyl 1,3-dithiolan2-ylidenemalonate) and 7.0\% flutolanil ( $\alpha, \alpha, \alpha$-trifluoro-3'isopropoxy-o-toluanilide) were used as pesticides. Twenty grams of soil slurry was taken in a $50 \mathrm{ml}$ of glass vial, and $10 \mathrm{ml}$ of distilled water was added to give a $20 \mathrm{~mm}$ layer of water on a $10 \mathrm{~mm}$ layer of soil. The recommended application rate, and 10, and 50 times the recommended rate of the pesticide formulations, which were determined based on the soil surface area in the vial, were added and mixed thoroughly with the soil. The actual amounts of the applied ZD and $\mathrm{MC}$ were $0.86 \mathrm{mg}$ and $3.4 \mathrm{mg}$ per vial, respectively, for the recommended application rate. Control samples without the application of pesticide were also prepared in the same way. Unflooded conditions were also prepared for some experiments without the addition of distilled water. The glass vial was covered with an aluminum foil sheet, and incubated at $25^{\circ} \mathrm{C}$ in darkness for four weeks. The accumulation of ferrous ion was observed under flooded conditions during the incubation period but not under unflooded conditions. Water was added periodically during the incubation period to compensate for evaporation. Each sample was prepared in duplicate.

\section{Biolog Patterns and Carbon Substrate Utilizing Activity of Soil Microbial Communities}

At one and four weeks after the pesticide treatment, the whole soil sample in one vial was taken and mixed with 170 $\mathrm{ml}$ of sterile distilled water to make 10 -fold serial dilutions. From the $10^{-3}$ dilution, $150 \mu 1$ aliquots were inoculated into each well of a GN type Biolog plate (Biolog Inc., CA), and incubated at $25^{\circ} \mathrm{C}$ for 4 days. Three plates were used for each sample. Color development in microplate wells was analyzed by measuring $A_{650}$ with a microplate reader (Wako model SPEDCTRA 1, Osaka, Japan) every 6-24 hr after the inoculation.

Average well color development (AWCD) $[\Sigma(\mathrm{C}-\mathrm{R}) / 95$, where $\mathrm{C}$ and $\mathrm{R}$ are absorbance values for wells with and without a sole carbon source, respectively] and positive well numbers $[(C-R)>0.1]$ were determined, and patterns of positive wells in the Biolog plates were grouped by cluster analysis (Ward linkage using squared Euclidean distance), using the SPSS for Windows software (ver. 7.5.1.0, SPSS Inc., IL, USA) according to a procedure described previously. ${ }^{7)}$ Biolog data at the time when almost half of the wells in each plate were positive were used for statistical analyses to avoid comparisons among plates in which the most and/or least number of wells were positive.

\section{RESULTS AND DISCUSSION}

Effect of Zark D5I and Fuji-One Moncut Powders on Soil Microbial Communities

Dendrograms of the Biolog patterns of soil microbial communities treated with and without $\mathrm{ZD}$ at the recommended dose and 50 times the application rate are shown in Fig. 1. No apparent differences between the control and ZD treatment at both rates and at one and four weeks after the herbicide treatment were observed. These results indicate that ZD had little effect on the soil microbial communities that are responsible for the Biolog color formation.

Dendrograms of the Biolog patterns of soil microbial communities treated with and without $\mathrm{MC}$ at the recommended and 50 times the recommended rate are shown in Fig. 2. At the recommended dose, the Biolog patterns between the control and MC-treated soil microbial communities could not be distinguished, however, at 50 times the application rate, the patterns were distinctly different between control and MC-treated soil microbial communities. These results indicate that $\mathrm{MC}$ had little effect on the soil (a)

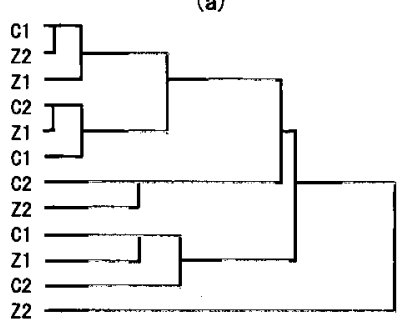

(c)

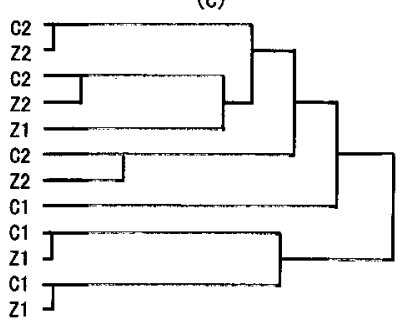

(b)

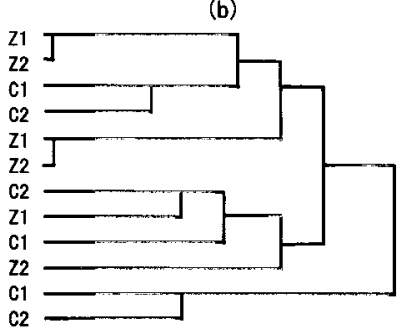

(d)

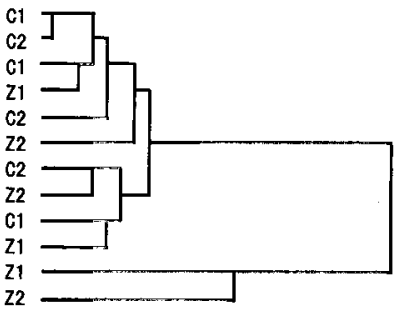

Fig. 1. Dendrograms of the Biolog patterns for microbial communities in paddy soils left untreated or treated with Zark D51 powder formulate.

(a) and (b), at one week after application of the herbicide at the recommended and 50 times the recommended rate, respectively; (c) and (d), at four weeks after the application at the recommended and 50 times the recommended rate, respectively. Hierarchial cluster analysis was conducted by the Ward linkage method using squared Euclidean distance. Labels are as follows; $\mathrm{Z}$, Zark D51 treatment; C, control; figures indicate duplicate samples, and three Biolog plates were used for each sample. 


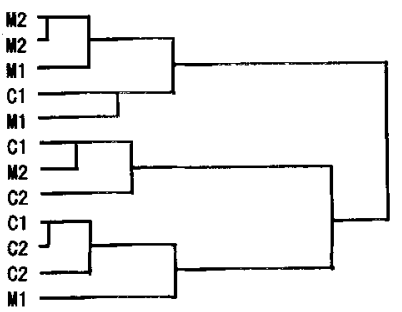

(c)

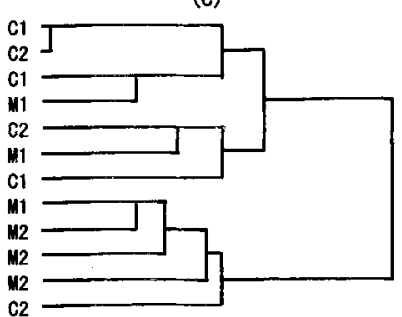

(b)

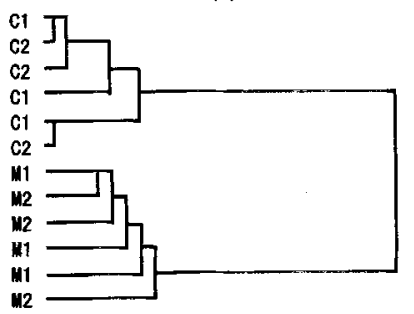

(d)

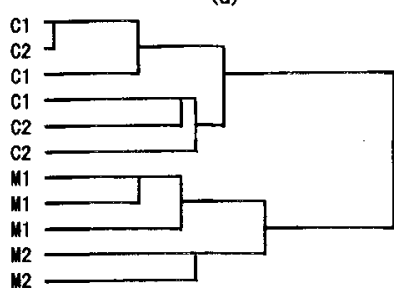

Fig. 2. Dendrograms of the Biolog patterns for microbial communities in paddy soils left untreated or treated with Fugi-One Moncut powder formulate.

(a) and (b), at one week after application of the fungicide at the recommended and 50 times the recommended rate, respectively; (c) and (d), at four weeks after the application at the recommended and 50 times the recommended rate, respectively. Hierarchial cluster analysis was conducted by the Ward linkage method using squared Euclidean distance. Labels are as follows; M, Fugi-One Moncut treatment; C, control; figures indicate duplicate samples, and three Biolog plates were used for each sample.

microbial communities at the recommended rate, but at 50 times this dose caused changes in soil microbial communities for at least four weeks.

(a)

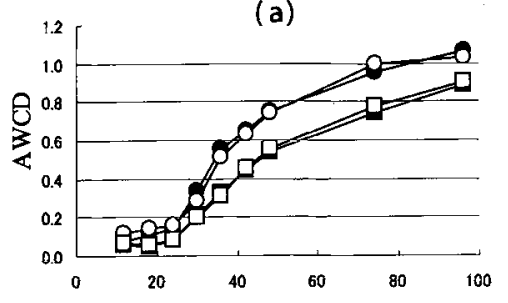

(c)

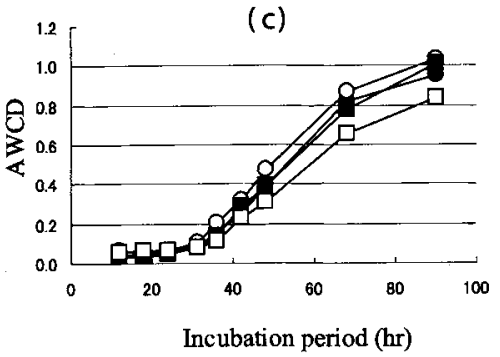

As one of the parameters of microbial activity, carbon substrate utilizing activity in the Biolog plate was compared between the control and pesticide-treated soil samples. Changes in AWCD and positive well numbers during incubation of the Biolog plates are shown in Fig. 3. As in the case of Biolog patterns, no significant difference from the control was observed in ZD-treated and MC-treated soil samples (data not shown). At 50 times the application rate of $\mathrm{MC}$, the level of activity was depressed at one week after the treatment, but the depression was not observed at four weeks. These results indicate that $\mathrm{MC}$ changed the soil microbial communities and depressed the activity at first, and then the activity recovered thereafter, but the microbial communities were still different from that of control.

Comparison of the Effects of Fuji-One Moncut Powder and Soil Flooding on Soil Microbial Communities

Significant changes in the Biolog patterns were observed on treatment with 50 times the recommended dose of MC. For assessing the significance of the changes in microbial communities, the magnitude of the change caused by $\mathrm{MC}$ should be compared with that caused by environmental parameters. If the magnitude of the change caused by $\mathrm{MC}$ is smaller than that caused by environmental parameters, the adverse effect of MC should be evaluated as insignificant because such changes usually take place in the field due to environmental stress. In our previous study, it was demonstrated that the flooding of paddy soil is one of the most significant parameters causing changes in the soil microbial communities determined by the Biolog method. ${ }^{6}$ Bossio and Scow also reported about different soil microbial communities in flooded and unflooded fields determined using phospholipid fatty acid profiles and carbon substrate utiliza-

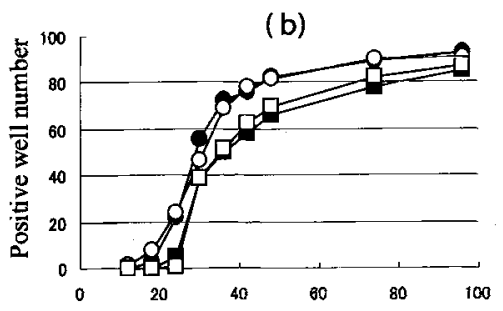

(d)

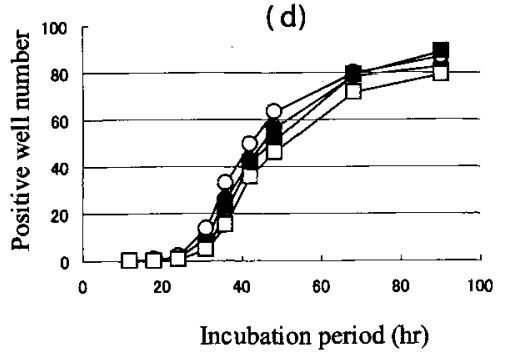

Fig. 3. Changes in average well color development (AWCD) and positive well number for microbial communities in paddy soils treated with Fugi-One Moncut powder formulate at 50 times the recommended rate.

(a) and (b), at one week after application of the fungicide; (c) and (d), at four weeks after the application of the fungicide. $\bullet$ and $\bigcirc$, control; $\square$ and $\square$, treatment of Fugi-One Moncut powder. 
(a)

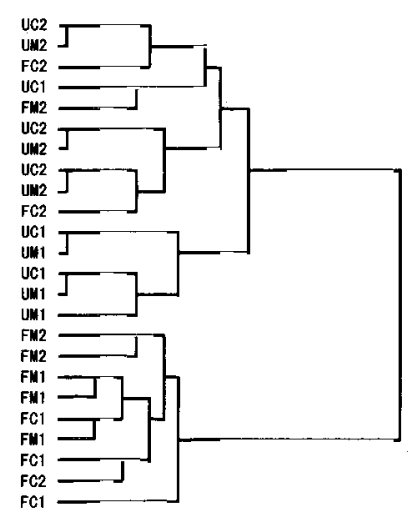

(b)

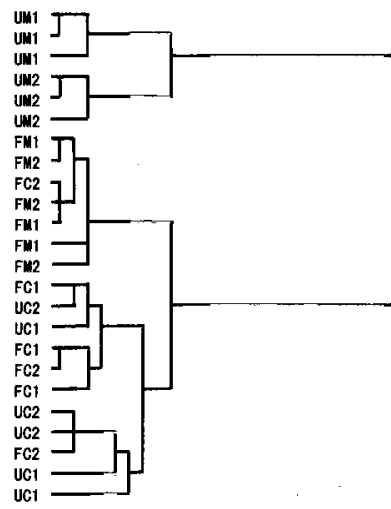

Fig. 4. Dendrograms of the Biolog patterns for microbial communities in paddy soils treated with Fugi-One Moncut powder formulate at 10 times the recommended rate.

(a) and (b), at one and four weeks after application of the fungicide, respectively. Hierarchial cluster analysis was conducted by the Ward linkage method using squared Euclidean distance. Labels are as follows; $\mathrm{U}$, unflooded conditions; $\mathrm{F}$, flooded conditions; M, Fugi-One Moncut treatment; C, control; figures indicate duplicate samples, and three Biolog plates were used for each sample.

tion patterns. ${ }^{8)}$ Therefore, the magnitude of the change in soil microbial communities caused by $\mathrm{MC}$ was compared with that caused by soil flooding. In this experiment, ten times the recommended dose of MC was applied.

As shown in Fig. 4 (a), the Biolog patterns of microbial communities at one week after MC treatment were mostly different between the flooded and unflooded soil samples regardless of the treatment. The results indicate that the characteristics of the microbial communities were determined mainly by flooding of the soil, not by the application of $\mathrm{MC}$.

On the other hand, at four weeks after the application, the microbial communities of the unflooded soil samples treated with $\mathrm{MC}$ were distinctly different from the other samples, as were those of flooded samples treated with MC (Fig. 4(b)). The untreated soil samples formed one cluster without the separation of flooded and unflooded soil samples. The results indicate that the treatment with MC caused changes in the microbial communities beyond the magnitude caused by the soil flooding. This means that such changes caused by $\mathrm{MC}$ are unlikely to occur under normal environmental conditions in the paddy soil.

Microorganisms play an important role in soil ecosystems, especially in nutrient cycles. Many studies have been conducted regarding the side-effects of pesticides on soil microbial populations, biomass and functions. ${ }^{1,2)}$ Soil microbial flora has been also considered to be an important

parameter for characterizing soil microbial ecosystems." The Biolog method has been applied to many environmental samples to differentiate microbial communities. ${ }^{10-12)}$ Changes in soil microbial communities caused by herbicides $^{13,14)}$ and fumigants ${ }^{15,16)}$ have been determined by the Biolog method, however, the level of significance of differences is a subject of concern. From an ecological point of view, the magnitude and duration of the effects of pesticides should be considered by comparing with those caused by natural stresses. ${ }^{3)}$ In this regard, we could detect changes in microbial communities even after the carbon source utilization activity was recovered, and could evaluate the significance of the changes by comparing the magnitude of that caused by the soil flooding which was the most influential environmental parameter acting on the microbial communities. As shown in this study, reasonable approaches are necessary for assessing the effects of pesticides on soil microbial ecosystems.

\section{REFERENCES}

1) J. R. Anderson: "Pesticide Microbiology," ed. by I. R. Hill and S. J. L. Wright, Academic Press, London, pp. 313-534, 1978.

2) "Pesticide Effects on Soil Microflora," ed. by L. Somerville and M. P. Greaves, Taylor and Francis, London, 1987.

3) K. H. Domsch, G. Jagnow and T. H. Anderson: Residue Rev. 86, 65-105 (1983).

4) Y. Okamoto, K. Suyama, K. Itoh, M. Itamochi, Y. Kagawa, S. Kajihara, T. Ikushima, H. Miyamoto and H. Yamamoto: $J$. Pesticide Sci. 24, 386-392 (1999).

5) K. Suyama, Y. Okamoto, K. Itoh, M. Itamochi, Y. Kagawa, K. Fujii, S. Kumagai, N. Koga, S. Kajihara, T. Ikushima, H. Miyamoto, M. Aoki, A. Kojima and H. Yamamoto: J. Pesticide Sci. 26, 127-135 (2001).

6) K. Itoh, T. Ikushima, K. Fujii, K. Suyama and H. Yamamoto: $J$. Pesticide Sci. 27, 360-364 (2002).

7) K. Itoh, D. Goto, K. Sueyasu, K. Suyama and H. Yamamoto: Soil Sci. Plant Nutri. 48, 333-339 (2002).

8) D. A. Bossio and K. M. Scow: Microbial Ecol. 35, 265-278 (1998).

9) "Beyond the Biomass," ed. by K. Ritz, J. Dighton and K. E. Giller, John Wiley and Sons, West Sussex, UK, 1996.

10) J. L. Garland and A. L. Mills: Appl. Environ. Microbiol. 57, 2351-2359 (1991).

11) J. L. Garland: FEMS Microbial. Eco1. 24, 289-300 (1997).

12) A. Konopka, L. Oliver and R. F. Turco Jr.: Microbial. Ecol. 35, 103-115 (1998).

13) S. E. Fantroussi, L. Verschuere, W. Verstraete and E. M. Top: Appl. Environ. Microbiol. 65, 982-988 (1999).

14) M. D. Busse, A. W. Ratcliff, C. J. Shestak and R. F. Powers: Soil Biol. Biochem. 33, 1777-1789 (2001).

15) K. Toyota, K. Ritz, S. Kuninaga and M. Kimura: Soil Sci. Plant Nutr. 45, 207-223 (1999).

16) A. M. Ibekwe, S. K. Papiernik, J. Gan, S. R. Yates, C. H. Yang and D. E. Crowley: Appl. Environ. Microbiol. 67, 3245-3257 (2001). 